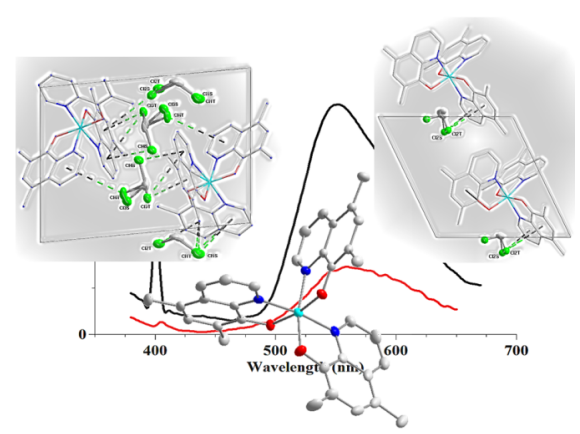

Figure 1. Crystallography as the decider in the optical behaviour of two gallium(III) complexes.

Keywords: Aluminium, Fluorescence, Quinolinol, Oleds

\section{MS21-P3 Phase transitions of silicon under dynamic and non-hydrostatic conditions}

Eva-Regine Carl $^{1}$, Jannik Richter ${ }^{2}$, Andreas N. Danilewsky ${ }^{2}$, Rajani K. Vijayaraghavan ${ }^{3}$, Sean Kelly ${ }^{3}$, Patrick McNally ${ }^{3}$, Zuzana Konopkova ${ }^{4}$, Hanns-Peter Liermann ${ }^{4}$

1. Institute of Earth and Environmental Sciences - Geology

2. Institute of Earth and Environmental Sciences - Crystallography

3. School of Electronic Engineering - Dublin City University

4. DESY

email: eva-regine.carl@geologie.uni-freiburg.de

Silicon represents one of the most important semiconductors in today's high-performance electronics. By the processing in the industry locally high pressures and temperatures emerge during the mechanical treatment and the wafer handling, leading to phase transitions under non-equilibrium conditions. This can result in a change of the electronic properties of the material, as well as the formation of cracks and the breaking of the wafers. We present time-resolved X-ray diffraction experiments under non-hydrostatic conditions that study in situ phase transformations of silicon. The experiments were conducted in a membrane-driven diamond anvil cell $(\mathrm{mDAC})$ at the Extreme Conditions Beamline (ECB) P02.2 at PETRA III, DESY. We used powered Si standard and added gold flakes for pressure determination. The compression rates varied around 0.04 $\mathrm{GPa} / \mathrm{s}$ and the maximal pressure went up to $20 \mathrm{GPa}$. Most experiments were conducted at room temperature. After one experiment, the sample was heated up to $500{ }^{\circ} \mathrm{C}$ using a graphite resistance heater. During compression and decompression, diffraction patterns were collected every 10 seconds. For analysis, three-dimensional contour plots were created to get an overview of the experiment to define the onset of phase transitions. In order to identify the phases and to determine the lattice parameters, we performed a LeBail analysis on single diffraction patterns. All experiments reveal two phase transitions during compression. Between 4 and $7 \mathrm{GPa}$, Si-I transforms to $\mathrm{Si}$-II. The next phase transition from $\mathrm{Si}$-II directly to Si-V occurs between 13 and $15 \mathrm{GPa}$, skipping the stability field of $\mathrm{Si}-\mathrm{XI}$. Si-V remains then stable to maximal pressure. Upon decompression, $\mathrm{Si}-\mathrm{V}$ transforms to $\mathrm{Si}$-III and $\mathrm{Si}-\mathrm{XII}$. The approximate proportion of $\mathrm{Si}$-III to $\mathrm{Si}-\mathrm{XII}$ is $20: 1$. The mixture remains stable to $2 \mathrm{GPa}$. At ambient pressure, this recovered sample was heated up to $500{ }^{\circ} \mathrm{C}$ to obtain Si-XIII. During heating, the two phases remain stable. Furthermore, there is no evidence that the proportion changes with respect to the diffraction patterns. However, a Raman-analysis of the recovered samples reveals a strong variation of this proportion over the entire sample. After maintaining at $500{ }^{\circ} \mathrm{C}$ for 5 minutes, two new reflections occur at low diffraction angles up to $11^{\circ} 2 \theta$ that can be indexed to Si-IV. However, two other strong reflections of this phase at higher 2 $\theta$-values are not observed. Being metastable, $\mathrm{Si}-\mathrm{IV}$ could not identified by a Raman-analysis.

Keywords: Silicon, non-hydrostatic 